\title{
Diminished Cortical Inhibition in an Aging Mouse Model of Chronic Tinnitus
}

\author{
Daniel A. Llano, ${ }^{1,2}$ Jeremy Turner, ${ }^{3,5}$ and Donald M. Caspary ${ }^{3,4}$ \\ ${ }^{1}$ Department of Molecular and Integrative Physiology and ${ }^{2}$ Beckman Institute, University of Illinois at Urbana-Champaign, Champaign, Illinois 61820, \\ Departments of ${ }^{3}$ Surgery/Otolaryngology and ${ }^{4}$ Pharmacology, Southern Illinois University School of Medicine, Springfield, Illinois 62702, and ${ }^{5}$ Department \\ of Psychology, Illinois College, Jacksonville, Illinois 62650
}

Flavoprotein autofluorescence imaging was used to examine auditory cortical synaptic responses in aged animals with behavioral evidence of tinnitus and hearing loss. Mice were exposed to noise trauma at 1-3 months of age and were assessed for behavioral evidence of tinnitus and hearing loss immediately after the noise trauma and again at $\sim 24-30$ months of age. Within 2 months of the final behavioral assessment, auditory cortical synaptic transmission was examined in brain slices using electrical stimulation of putative thalamocortical afferents, and flavoprotein autofluorescence imaging was used to measure cortical activation. Noise-exposed animals showed a $68 \%$ increase in amplitude of cortical activation compared with controls $(p=0.008)$, and these animals showed a diminished sensitivity to GABA $\mathrm{A}_{\mathrm{A}}$ ergic blockade $(p=0.008$, using bath-applied $200 \mathrm{~nm}$ SR 95531 [6-Imino-3-(4-methoxyphenyl)-1(6H)- $\mathrm{p}$ yridazinebutanoic acid hydrobromide]). The strength of cortical activation was significantly correlated to the degree of tinnitus behavior, assessed via a loss of gap detection in a startle paradigm. The decrease in $\mathrm{GABA}_{\mathrm{A}}$ sensitivity was greater in the regions of the cortex farther away from the stimulation site, potentially reflecting a greater sensitivity of corticocortical versus thalamocortical projections to the effects of noise trauma. Finally, there was no relationship between auditory cortical activation and activation of the somatosensory cortex in the same slices, suggesting that the increases in auditory cortical activation were not attributable to a generalized hyperexcitable state in noise-exposed animals. These data suggest that noise trauma can cause long-lasting changes in the auditory cortical physiology and may provide specific targets to ameliorate the effects of chronic tinnitus.

\section{Introduction}

Tinnitus is a common and often disabling symptom that increases in prevalence with age. It is characterized by the presence of abnormal spontaneous auditory percepts and is often associated with other symptoms, such as hyperacusis. Most population studies place the prevalence of tinnitus at 5-20\%, with the proportion of those having moderate to severe symptoms being $20-$ $30 \%$ (Møller, 2011). Tinnitus is almost always associated with peripheral hearing loss. This has led to the hypothesis that damage to peripheral auditory structures leads to central reorganization and that this maladaptive reorganization may lead to tinnitus. Several specific mechanisms for tinnitus have been proposed, including pathological synchronous cortical activation (Eggermont, 2007), homeostatic synaptic plasticity (Yang et al., 2011), or diminished synaptic inhibition (Wang et al., 2011).

The finding that tinnitus may be caused by damage to peripheral hearing structures has led to the development of several dif-

\footnotetext{
Received May 21, 2012; revised Aug. 21, 2012; accepted Sept. 11, 2012.

Author contributions: D.A.L., J.T., and D.M.C. designed research; D.A.L. and J.T. performed research; J.T. contributed unpublished reagents/analytic tools; D.A.L., J.T., and D.M.C. analyzed data; D.A.L. wrote the paper.

This work was supported by the National Institute on Deafness and Other Communication Disorders (D.A.L., D.M.C.) and the Tinnitus Research Consortium (J.T.). We thank Deb Larsen and Alexandria Lesicko for their expert technical assistance.

Correspondence should be addressed to Daniel A. Llano, 2355 Beckman Institute, 405 North Mathews Avenue, Urbana, IL 61801. E-mail: d-llano@illinois.edu.

DOI:10.1523/JNEUROSCI.2499-12.2012

Copyright $\odot 2012$ the authors $\quad 0270-6474 / 12 / 3216141-08 \$ 15.00 / 0$
}

ferent animal models. These models typically involve a lesion of the peripheral hearing apparatus (Kaltenbach, 2011), and studies in these tinnitus models have revealed increases in spontaneous and driven activity seen in cochlear nucleus (Kaltenbach et al., 1998; Brozoski et al., 2002; Middleton et al., 2011), inferior colliculus (Willott and Lu, 1982; Salvi et al., 1990; Szczepaniak and Møller, 1995), and auditory cortex (Yang et al., 2011). These findings are consistent with the notion that peripheral hearing loss causes an increase in excitability in central auditory structures, possibly to enhance the processing of weakened peripheral sensory input (Wang et al., 2011). Unfortunately, most animalbased studies in the literature have used relatively young animals and studied the development of tinnitus in the acute or subacute phase after injury. However, tinnitus is most commonly encountered in the elderly population and frequently persists for many years. Therefore, in the current study, the functional consequences of chronic tinnitus in an aging population of mice were examined.

Although multiple central auditory structures have been implicated in tinnitus, recent findings point to a potential role for the auditory cortex (Mühlnickel et al., 1998; van der Loo et al., 2009; Noreña et al., 2010; Schecklmann et al., 2011; Stolzberg et al., 2011; Yang et al., 2011). Understanding the precise mechanisms underlying changes in auditory cortical processing mechanisms during tinnitus has important therapeutic implications because of recent attempts to treat tinnitus via direct modulation of auditory cortical excitability (Kleinjung et al., 2008). One dif- 
ficulty in studying central mechanisms of tinnitus has been the separation of peripheral versus central changes in synaptic organization. Brain slice preparations may therefore be useful because they allow the isolation of potentially affected subcircuits within the auditory system.

Here, we examined the earliest level of cortical processing by studying the effects of stimulating afferents to the auditory cortex in animals with behavioral evidence of tinnitus. Auditory cortical activation was measured using flavoprotein autofluorescence, which permits the measurement of neural activity with high sensitivity and spatial resolution, without the potentially confounding factors present when using exogenous dyes, such as differential uptake or dye toxicity (Shibuki et al., 2003; Llano et al., 2009). In addition, given evidence for GABAergic (Wang et al., 2011) and NMDA (Lobarinas et al., 2006; Hwang et al., 2011) dysfunction in tinnitus, pharmacologic manipulations of these systems was also done.

\section{Materials and Methods}

Mice. A total of 13 CBA/CAj male mice were used in this study. Six mice were exposed to closed-field unilateral noise stimulus, four mice were given sham treatment, and three mice did not receive sham treatment but were matched for age with the rest of the cohort. The data for the shamtreated and untreated older mice were similar and were pooled. All procedures used in the research protocol were approved by the Southern Illinois University School of Medicine and the University of Illinois Laboratory Animal Care and Use Committees (protocols \#P190-06-005 and \#10208, respectively), conformed to the National Institutes of Health Guide for the Care and Use of Laboratory Animals, and followed the Society for Neuroscience Guidelines for the Use of Animals in Neuroscience Research.

Noise trauma and behavioral testing. Mice were pretested using the gap prepulse inhibition (PPI) of acoustic startle procedure (Turner et al., 2006) and placed into either noise trauma or control (sham trauma) groups. The noise trauma consisted of a peak-calibrated level of $116 \mathrm{~dB}$, centered on a $16 \mathrm{kHz}$ octave band. The noise exposure was presented unilaterally to isoflurane-anesthetized mice for $1 \mathrm{~h}$. Mice were of average age of $66 \mathrm{~d}$ at the time of noise exposure or sham.

Behavioral testing was conducted using startle reflex hardware and software, customized for this application by the manufacturer (Kinder Scientific). Background sounds in the startle chamber consisted of $60 \mathrm{~dB}$ SPL, $1000 \mathrm{~Hz}$ bandpass filtered noise ( $48 \mathrm{~dB}$ /octave roll off; Krohn-Hite model 3988) centered at 4, 8, 10, 12.5, 16, 20, 24, and $32 \mathrm{kHz}$ and broadband noise. Gap and PPI testing used background sounds presented through one speaker (Vifa XT25TG30-04) and startle stimuli presented through a second speaker (Powerline CTS KSN-1005) located in the ceiling of the testing chamber, $15 \mathrm{~cm}$ above the animal's head. PPI was measured as a control condition to assess for hearing loss. Prepulse stimuli matched the backgrounds used in gap testing in both spectral and intensity dimensions. For schematic of PPI sound sequence, see Figure $1 \mathrm{~A}$. PPI responses were expressed as the ratio of the motor response to the prepulse to the motor response of the startle pulse, where 1.0 equals no PPI (suggesting loss of the ability to detect the prepulse). Gap testing was used to assess behavioral evidence of tinnitus. For schematic of the gap testing sequence, see Figure $1 B$. Gap ratio equals the ratio of the response to the startle stimulus without gap, to the startle stimulus with a preceding gap. Because animals with tinnitus perceive sound during the gap period, they will show reduction in the inhibition of the startle normally cued by the gap (i.e., the gap ratio will approach 1.0 ). Occasionally, values $>1.0$ are seen, and these are likely related to the fact that the technique has a floor of zero but no ceiling, coupled with behavioral variability seen in the technique. A specific value for the gap ratio was not used to assign mice to belonging to a "tinnitus" group (see below, Analysis). The use of gap-induced reduction of startle for the assessment of tinnitus has been validated against conditioning-based approaches to tinnitus and has been shown to be a reliable metric across several species (Turner, 2007; Longenecker and Galazyuk, 2011; Turner et al., 2012).
Auditory brainstem response thresholds. Auditory brainstem response (ABR) thresholds were obtained for each ear immediately before and after unilateral noise exposure. However, exit ABRs were not obtained on all animals because several animals died during ABR testing, likely related to their old age and use of anesthesia at the time of testing.

Brain slicing. We used a slight modification of the thalamocortical brain slice preparation developed by Cruikshank et al. (2002). This preparation was used to increase the likelihood of capturing thalamocortical afferents to the auditory cortex. Chemoarchitectonic criteria (see Fig. 2), suggests that the imaging data were derived from primary auditory fields (primary auditory cortex and/or anterior auditory field). Use of the term "auditory cortex" within this report refers to primary auditory fields and makes no attempt to distinguish between primary auditory cortex and anterior auditory field.

Brain slices were obtained containing thalamocortical projections contralateral to the ear that received noise trauma. In brief, mice were initially anesthetized with a ketamine $(1 \mathrm{mg} / \mathrm{g}) /$ xylazine $(10 \mathrm{mg} / \mathrm{g})$. Mice were then perfused with chilled $\left(4^{\circ} \mathrm{C}\right)$ sucrose-based slicing solution (in mM): 234 sucrose, 11 glucose, $26 \mathrm{NaHCO}_{3}, 2.5 \mathrm{KCl}, 1.25 \mathrm{NaH}_{2} \mathrm{PO}_{4}, 10$ $\mathrm{MgCl}_{2}$, and $0.5 \mathrm{CaCl}_{2}$. The brain was blocked by removing the olfactory bulbs and the anterior $2 \mathrm{~mm}$ of frontal cortex with a razor blade. The brain was then placed on its cut surface, and an off-horizontal cut was made from the dorsal surface. This cut was angled at $20^{\circ}$ from the horizontal plane such that a small dorsal flap was removed. The brain was then glued using the newly cut surface onto a cutting platform, and $600-\mu \mathrm{m}$-thick sections were cut. Sections were allowed to incubate at $32^{\circ} \mathrm{C}$ for $1 \mathrm{~h}$ before use in an incubation solution $\left(26 \mathrm{NaHCO}_{3}, 2.5 \mathrm{KCl}\right.$, 10 glucose, $126 \mathrm{NaCl}, 1.25 \mathrm{NaH}_{2} \mathrm{PO}_{4}, 3 \mathrm{MgCl}_{2}$, and $1 \mathrm{CaCl}_{2}$ ). For flavoprotein experiments, slices were transferred to a chamber perfused with ACSF $\left(26 \mathrm{NaHCO}_{3}, 2.5 \mathrm{KCl}, 10\right.$ glucose, $126 \mathrm{NaCl}, 1.25 \mathrm{NaH}_{2} \mathrm{PO}_{4}, 1$ $\mathrm{MgCl}_{2}$, and $3 \mathrm{CaCl}_{2}$ ) at room temperature, bubbled with $95 \%$ oxygen $/ 5 \%$ carbon dioxide. The experimenter was blinded to the status of the animals until after the experiment was completed.

Stimulation and flavoprotein imaging. Glass micropipettes were broken back to a diameter of $50 \mu \mathrm{m}$, filled with ACSF, and gently placed onto a fiber bundle $\sim 100-200 \mu \mathrm{m}$ ventral to the auditory cortex. The stimulating electrode was placed in the subcortical white matter rather than the thalamus because, in our experience and that of others (Broicher et al., 2010), there is a great deal of slice-to-slice variability in the ability to capture intact projections from thalamus to cortex. This would make it difficult in a relatively small sample size to see differences between noiseexposed and control animals. In several experiments, the stimulating electrode was moved $2 \mathrm{~mm}$ rostrally to the white matter beneath the secondary somatosensory cortex (Franklin and Paxinos, 2007) to determine the specificity of the findings to the auditory cortex. Trains of electrical pulses were delivered using PowerLab software and hardware and a World Precision Instruments stimulus isolator. Trains were $1 \mathrm{~s}$ in duration, with $2 \mathrm{~ms}$ pulses, delivered at 40 pulses/s, and trains were delivered every $20 \mathrm{~s}$, with five repetitions total.

Flavoprotein autofluorescence was used to measure cortical activation. This technique takes advantage of the endogenous fluorescence of mitochondrial flavoproteins. These molecules fluoresce when oxidized and will cause neuronal tissue to fluoresce when active. Flavoprotein autofluorescence has been shown to be a highly stable, sensitive, and specific marker for neuronal activation, both in vivo and in vitro (Shibuki et al., 2003; Husson et al., 2007; Llano et al., 2009; Reinert et al., 2011). A fluorescence illuminator (either a Prior Lumen 200 or Olympus X-Cite Series $120 \mathrm{PC}$ Q) and a UMNIB Olympus filter cube (470-490 nm excitation, $505 \mathrm{~nm}$ dichroic, $515 \mathrm{~nm}$ emission long pass) was used for fluorescence optics. A coverslip was placed over the slice to provide a stable imaging plane. All data were collected at four frames per second using an infinity-corrected $4 \times$ objective (NA 0.13 ) and a Retiga EXi camera using $4 \times 4$ binning and StreamPix software.

Drugs. Two hundred nanomolar SR 95531 [2-(3-carboxypropyl)-3amino-6-(4-methoxyphenyl)pyridazinium bromide], $50 \mu \mathrm{M}$ APV, 100 $\mu \mathrm{M}$ DNQX, and $1 \mu \mathrm{M}$ tetrodotoxin were all purchased from Tocris Bioscience. In all cases, drugs were dissolved in ACSF just before the experiment, and $1 \mathrm{~h}$ wash-in and $1 \mathrm{~h}$ washout times were used.

Histology. Cytochrome oxidase staining, parvalbumin immunohistochemistry, and Nissl staining were used to assist in identifying imaged 
regions in brain slices. Previous work suggested that these markers separate primary auditory fields (primary auditory cortex and anterior auditory field) from nonprimary fields (Caviness, 1975; Wallace et al., 1991; Molinari et al., 1995; Budinger et al., 2000; Cruikshank et al., 2001; Llano and Sherman, 2008) Slices were stored in $4 \%$ paraformaldehyde in PBS after each experiment. They were then placed in an ascending sucrose gradient up to $30 \%$ sucrose in PBS, frozen, and resectioned on a freezing microtome at $75 \mu \mathrm{m}$. For immunostaining, sections were washed in PBS, placed in a microwave for $15 \mathrm{~s}$ to increase antigenicity, and then incubated for $30 \mathrm{~min}$ in PBS containing 0.3\% Triton X-100, followed by $3 \%$ normal goat serum (NGS). Sections were then incubated with primary antibody solution consisting of monoclonal anti-parvalbumin produced in mouse [1:1000, catalog \#P3088 (Sigma), in 0.3\% Triton X-100 and 3\% NGS in PBS]. Secondary antibody consisted of 1:100 goat anti-mouse IgG coupled to Alexa Fluor 488 (catalog \#A-11001; Invitrogen), to produce green fluorescence. For cytochrome oxidase staining, the method of Wong-Riley (1979) was used. Sections were incubated with a solution of $0.05 \%$ DAB, $4 \%$ sucrose, and $20 \%$ cytochrome $c$ (Sigma) in PBS in the dark at $37^{\circ} \mathrm{C}$ for $4 \mathrm{~h}$ and quenched with PBS.

Analyses. For behavioral data, two complementary types of analysis were done. First, a categorical analysis based on noise-exposure or noexposure/controls was done. No attempt was made to segregate animals based on the presence or absence of tinnitus. Second, a correlation analysis was done to assess whether a quantitative relationship exists between tinnitus-related deficits and cortical activation measurements. This was done because it is likely that some noise-exposed animals may not develop tinnitus and because some older mice (like some older humans) may spontaneously develop hearing loss and tinnitus. Here, all animals were pooled, and no assumptions were made about the development of tinnitus based on group assignment.

For imaging data, in each run, a total of 420 images were collected, and image size was $260 \times 348$ pixels (using $4 \times 4$ hardware binning). The first $5 \mathrm{~s}$ (20 frames) were used as a baseline for the subsequent images. All image analysis, except curve fitting, was done using custom software written in MATLAB. Time-based analyses were done by computing change in fluorescence as a function of baseline fluorescence. Time series for each pixel were corrected for quenching by high-pass filtering each time series. Several regions of interest were used for analysis. An "onbeam" region of interest was constructed centered on the column of activation seen without blockade of $\mathrm{GABA}_{\mathrm{A}}$ receptors (see Fig. 2). For this, a $1 \mathrm{~mm}$ (rostral-caudal) region of interest from the pial surface to the gray-white junction was used, and a change in fluorescence was computed, relative to the first $5 \mathrm{~s}$ ( 20 frames) of the run. An additional region of interest, termed the "off-beam" region, was of same width of the above-described on-beam region $(1 \mathrm{~mm})$ but was displaced caudally from the on-beam region and did not overlap the on-beam region. This off-beam regions corresponds to most of the remainder of the primary auditory cortex not encompassed in the on-beam region (Cruikshank et al., 2002; Hackett et al., 2011). For each stimulation site, a series of stimulation amplitudes were used, ranging from 5 to $500 \mu \mathrm{A}$, and an inputoutput curve was constructed. In all cases, input-output curves resembled a sigmoidal function, and therefore a traditional fourparameter dose-response curve using ReaderFit software was used to fit the data. From the fit curves, the minimum, maximum, midpoint $\left(\mathrm{EC}_{50}\right)$, and slope could be extracted.

To compute the width of activation, a region of interest comprising the whole cortex was used. Images from runs at saturation stimulus amplitudes were imported into NIH ImageJ (http://rsbweb.nih.gov/ij/), and the curvature of the cortex was corrected using the "Straighten" function in NIH ImageJ. NIH ImageJ was then used to collapse all pixel values from pia to the white matter into a linear array of bins, and this histogram was used to compute a full-width at half-maximum.

For image display, spatial averaging was done using a $3 \times 3$ kernel. Change in fluorescence over baseline fluorescence maps ( $\Delta f / f$ maps) were overlaid on raw fluorescence images for localization of anatomic structures.

Statistics. Given the relatively small numbers of animals in each group, nonparametric statistics were used. To compare across groups (control vs noise-exposed), a Mann-Whitney $U$ test statistic was used, and, for

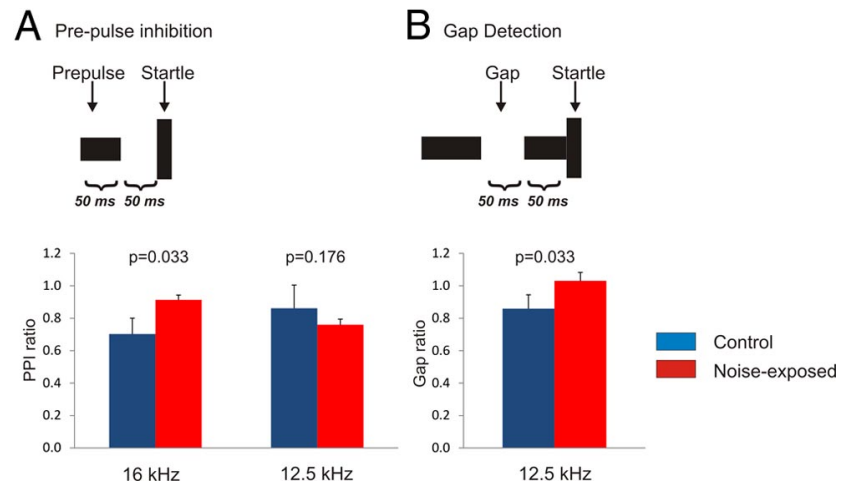

Figure 1. Behavioral assessment of hearing loss and tinnitus behavior in noise-exposed animals. The top images illustrate temporal sequences of sounds used to assess $\operatorname{PPI}(\boldsymbol{A})$ and gap detection $(\boldsymbol{B})$. Bar graphs on the left illustrate the mean and SE of the PPI ratio (defined in Materials and Methods) of control animals $(n=4)$ and noise-exposed animals ( $n=6)$. Bar graphs on the right illustrate mean and SE values for the gap detection ratio of control animals $(n=4)$ and noise-exposed animals $(n=6)$. $p$ values are derived from Mann-Whitney $U$ test.

correlation analysis, a Spearman's correlation was calculated. $p<0.05$ was taken as an indicator of statistical significance. Two-tailed testing was done for all analysis except for behavioral testing (PPI ratio and gap ratio), in which one-tailed testing was done. This is because responses are indexed against a theoretical minimum of zero (for PPI) or theoretical maximum of 1.0 (for gap), and only deviations upward (for PPI) or downward (for gap) are of relevance. Mann-Whitney test and Spearman's correlations were run using GraphPad software. To assess interactions between noise-exposure and location in auditory cortex (on-beam vs off-beam) or layer of cortex (supragranular vs infragranular), a mixedeffects model was used. Data were log transformed to maintain normality, and post hoc analysis was performed to assess for an interaction of condition by brain region. Mixed-effects modeling was done in $\mathrm{R}$ (http://www.r-project.org/).

\section{Results}

Thirteen aged mice were used in this study. Six animals were exposed to noise trauma, four animals were exposed to sham conditions, and three additional age-matched animals were included in the control dataset. The mean \pm SD age at the time that thalamocortical slices were cut did not significantly differ in the two groups (controls, $26.7 \pm 3.6$ months; tinnitus animals, $28.6 \pm 2.6$ months; $p=0.285$ ).

Animals were assessed for hearing loss at an average \pm SD of $24.7 \pm 1.7$ months after the noise exposure or sham. At $16 \mathrm{kHz}$, noise-exposed mice demonstrated an increase in PPI ratio relative to controls (Fig. 1A), suggesting that these noise-exposed animals had hearing loss at this frequency. There was no increase in PPI ratio at $12.5 \mathrm{kHz}$; therefore, $12.5 \mathrm{kHz}$ frequency was used for assessment of gap detection. An increase in gap ratio was observed in the noise-exposed animals at $12.5 \mathrm{kHz}$ (Fig. $1 B$ ), suggesting that these animals had behavioral evidence of tinnitus. No significant differences in gap ratio were seen between groups when tested at frequencies from 4 to $10 \mathrm{kHz}$ or from 20 to $31 \mathrm{kHz}$.

Cortical responses were measured in all animals. In all cases, electrical stimulation of the white matter ventral to the cortex produced a robust change in fluorescence in a column of the overlying auditory cortex (Fig. 2A,B). Exposure of the slices to $200 \mathrm{nM}$ SR 95331 caused a significant increase in the magnitude and breadth of the cortical response to axonal stimulation (Fig. $2 C, D)$. The concentration of $200 \mathrm{~nm}$ SR 95331 was chosen because, in this preparation, we have found that concentrations higher than $500 \mathrm{~nm}$ often lead to paroxysmal activity. The in- 
crease in breadth of the response in all tissues was anisotropic, that is, was only seen extending into the caudal direction.

Under conditions of normal ACSF, the peak of activation occurred within $\sim 2$ s of the stimulus onset and then gradually returned to baseline over the subsequent 10-20 s. As shown in Figure 2E, the peak in the response dropped by $>85 \%$ in response to blockade of ionotropic glutamate receptors using $100 \mu \mathrm{M}$ APV and 80 $\mu \mathrm{M}$ DNQX and dropped by $>95 \%$ with the use of tetrodotoxin, suggesting that the changes in cortical fluorescence are caused by axonal stimulation and that the majority of the response is driven by orthodromic and likely thalamocortical stimulation. In all cases, gradual increases of stimulation amplitude from 5 to 500 $\mu \mathrm{A}$ produced a nonlinear increase in response amplitude that resembled sigmoidal response function (Fig. 2F). Nissl staining (Fig. 2G) and immunostaining for parvalbumin (Fig. $2 H$ ) of different sections taken from the same slice reveal that the area of flavoprotein activation corresponds to an area of cortex with granularity in layer 4 and strong parvalbumin immunopositivity relative to adjacent regions, respectively. Based on these findings and previous work (Cruikshank et al., 2002; Hackett et al., 2011), this area likely corresponds to the primary auditory cortex.

Cortical response magnitude differed between the control animals and the noise-exposed animals. Examples of cortical activation are shown in Figure 3, A and $B$, demonstrating a substantially greater response in the noise-exposed animal. As shown in Figure $4 A$, the mean \pm $\mathrm{SD}$ on-beam response to afferent stimulation in control animals was $1.9 \pm 0.5 \%$ $(n=7)$ above baseline, whereas in noiseexposed animals, this increase was $3.2 \pm$ $0.8 \%(n=6 ; p=0.008$, Mann-Whitney $U$ test), yielding a mean increase in response amplitude of $68 \%$. Blockade of $\mathrm{GABA}_{\mathrm{A}}$ receptors with $20 \mathrm{~nm}$ SR 95531 increased the strength of the responses in both groups, such that the responses under conditions of $\mathrm{GABA}_{\mathrm{A}}$ blockade were not significantly different (mean $\pm \mathrm{SD}, 4.29 \pm 1.36 \%$ vs $3.48 \pm 0.96 \% ; p=0.29$, Mann-Whitney $U$ test). Sensitivity of this response to $\mathrm{GABA}_{\mathrm{A}}$ blockade was assessed using the ratio of the cortical response under conditions of $\mathrm{GABA}_{\mathrm{A}}$ blockade to conditions when normal ACSF was used. For control animals, the mean $\pm \mathrm{SD}$ ratio was $1.86 \pm 0.33(n=7)$, whereas in noiseexposed animals, this ratio decreased to a mean \pm SD value of $1.32 \pm 0.22(n=6 ; p=0.008$, Mann-Whitney $U$ test; Fig. $4 B)$. Full-width at half-maximum trended higher in noise-exposed animals than control animals (mean \pm SD, $1215 \pm 371$ vs $1500 \pm$ $223 \mu \mathrm{m} ; p=0.13$ ) but did not reach the 0.05 threshold.

The off-beam regions of cortex were analyzed for strength of activation and sensitivity to SR 95531. Analysis of the strength of activation in these zones in animals revealed that noise-exposed animals had a significantly greater activation by greater than twofold compared with control animals (Fig. 4C; mean $\pm \mathrm{SD}$, control, $0.62 \pm 0.23 \%$; tinnitus, $2.1 \pm 0.9 \% ; p=0.003$, Mann-Whitney $U$ test). In addition, noise-exposed animals demonstrated significantly less sensitivity to SR 95531 than controls (Fig. 4D; mean \pm SD ratio of response under SR 95531 conditions vs normal ACSF in control animals, $5.45 \pm 2.4$ and tinnitus animals, $1.82 \pm 0.49 ; p=0.003$, Mann-Whitney $U$ test). In addition, in a mixed-effects model analysis, condition $\times$ brain region interaction showed a significant effect of brain region on the difference between tinnitus and control animals for both amplitude of activation $(p=0.01)$ and sensitivity to SR $95531(p=0.01)$.

In addition, increases in cortical activation were seen in noiseexposed animals in both the infragranular (1.69 vs $2.67 \%$; $p=$ $0.022)$ and supragranular (2.11 vs $4.43 \% ; p=0.001)$ layers (Fig. 

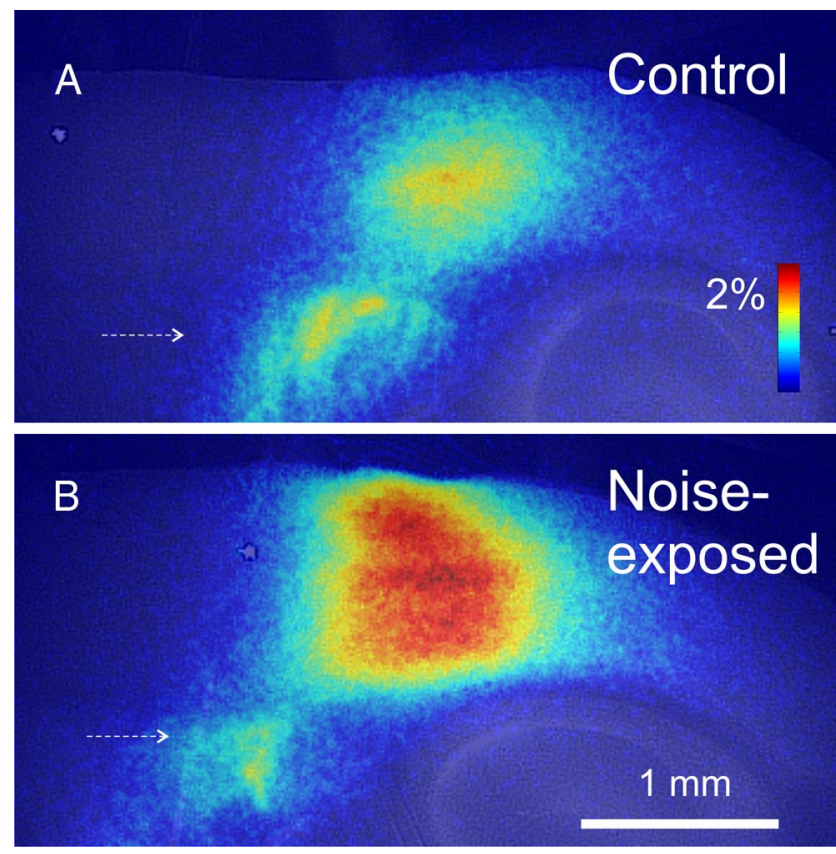

Figure 3. Examples of $\Delta f / f$ cortical activation maps for a control animal $(\boldsymbol{A})$ and a noiseexposed animal (B). Stimulus amplitudes/parameters were identical: $300 \mu \mathrm{A}, 2 \mathrm{~ms}$ duration pulses at 40 pulses $/ \mathrm{s}$ for $1 \mathrm{~s}$. Color bar corresponds to the percentage increase in fluorescence over baseline.

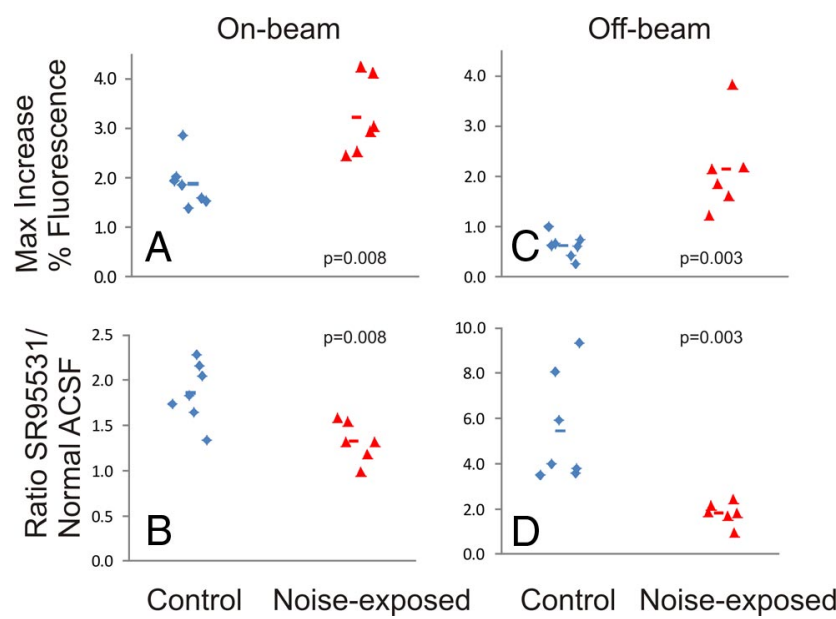

Figure 4. Comparison of strength of cortical activation and $\mathrm{GABA}_{\mathrm{A}} \mathrm{ergic}$ sensitivity in the on-beam and off-beam regions of the auditory cortex (left and right, respectively). Top row shows the maximum increase in fluorescence. Bottom row shows the ratio of the response under conditions of exposure to $200 \mathrm{~nm}$ SR 95331 to normal ACSF. Each diamond or triangle represents a single slice. Horizontal lines represent mean value. $P$ values are derived from Mann-Whitney $U$ test.

5). An interaction between noise exposure and cortical layers was observed, such that there was a greater increase in the supragranular layers (mixed-effect model, $p=0.01$ ).

Slices from 11 animals (six controls and five noise-exposed) were exposed to $100 \mu \mathrm{M}$ APV to assess the effects of NMDA blockade on cortical activation. APV induced a modest drop in the cortical response that did not differ between control versus noise-exposed animals (mean drop of 27 vs $22 \%$ in control vs noise-exposed; $p=0.646$, Mann-Whitney $U$ test; Fig. 6).

To assess whether the increase in auditory cortical responsiveness was attributable to a generalized increase in excitability ver-

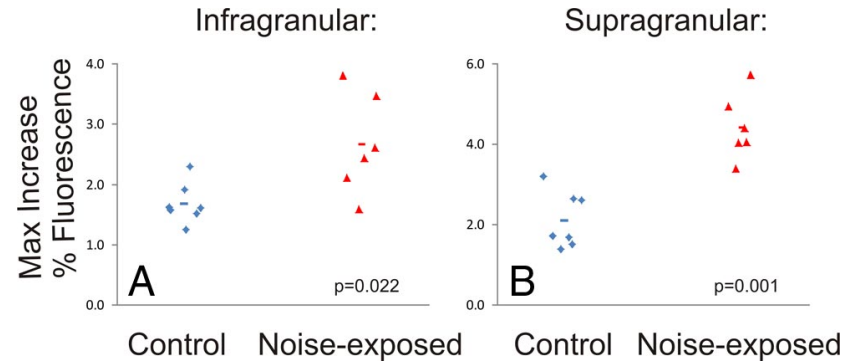

Figure 5. Comparison of strength of cortical activation in the infragranular and supragranular layers of the auditory cortex ( $\boldsymbol{A}$ and $\boldsymbol{B}$, respectively). Each diamond or triangle represents a single slice. Horizontal lines represent mean value. $P$ values are derived from Mann-Whitney $U$ test.

sus a specific change in the auditory cortical system, responses in the auditory cortex were compared with responses in the somatosensory cortex. Identical analysis protocols were used for the two cortical areas. The stimulation site for the somatosensory cortex was $\sim 2 \mathrm{~mm}$ rostral to the site for the auditory cortex (Fig. $7 A$ ). This area of cortex does not show barrel architecture, confirming that this is non-barrel cortex (Fig. 7B). An example of a flavoprotein response in the somatosensory cortex is shown in Figure 7C. In general, somatosensory responses were smaller than those in the auditory cortex, but the response magnitudes were uncorrelated (Fig. $7 D ; R^{2}=0.51, p=0.154, n=6$ ), suggesting that changes described above in the auditory cortex were not attributable to a generalized change in cortical excitability.

Correlations between behavioral measures of hearing loss and tinnitus, quantified using a paired-pulse protocol and gap detection protocol, respectively, and cortical responses, were assessed. There was a significant $(p=0.024)$ trend for a positive association between gap detection ratio at $12.5 \mathrm{kHz}$ and cortical response magnitude (Fig. $8 A ; R^{2}=0.52, p=0.024$ ). There was a nonsignificant trend for a positive correlation between the PPI ratio at $12.5 \mathrm{kHz}$ and cortical response magnitude (Fig. $8 B ; R^{2}=$ $0.31, p=0.081)$.

\section{Discussion}

In the current study, we show that aged animals with noise exposure in young adulthood and subsequent behavioral evidence of hearing loss and tinnitus demonstrate changes in auditory cortical responses to afferent stimulation. Specifically, noise-exposed animals show a greater magnitude of auditory cortical activation and show a diminished sensitivity to $\mathrm{GABA}_{\mathrm{A}}$ blockade than control animals. These findings appeared to be more prominent in the upper cortical layers, specific to the auditory cortex (rather than the somatosensory cortex), and other receptor systems, such as the NMDA system, did not appear to be altered in tinnitus animals in this preparation.

\section{Methodological considerations}

The current study is limited by the blind approach to stimulation and imaging in the auditory thalamocortical slice preparation. Although noise damage was induced at a particular range of frequencies ( 1 octave band centered at $16 \mathrm{kHz}$ ), the frequency representation of the stimulated area of cortex in the thalamocortical slice preparation is not known. Although it is possible to map frequency representations before slicing using either electrophysiological means (Hackett et al., 2011) or non-invasive imaging (Takahashi et al., 2006), this was not attempted here given the overall frailty of these aged animals. It should be noted that, despite this limitation, we did observe significant changes in the 
blind activations of the auditory cortex, similar to changes seen with blind stimulation of the afferents to the cochlear nucleus (Middleton et al., 2011). Future work will involve auditory cortical mapping before slice preparation to better understand the relationship between the spectral characteristics of noise exposure and alterations in cortical activations in relation to the tonotopic map of the auditory cortex.

It should be noted that the specific circuitry driving the cortical activation signal is not known. Given the substantial drop $(>85 \%)$ in cortical activation after blockade of NMDA and AMPA receptors (Fig. $1 E)$, similar to our previous work in the somatosensory thalamocortical slice (Llano et al., 2009), we assume that most of the signal is driven by orthrodromic activation of thalamocortical afferents. However, there are other circuit elements, such as shortrange corticocortical activations, that may be responsible for the fluorescence signal and may contribute to the differences seen between control and tinnitus animals.

\section{Mechanisms underlying \\ noise-induced changes}

The specific behavioral consequence of noise exposure that is associated with the changes in cortical responses described here is not known. Animals in this study demonstrated evidence for both hearing loss (evidenced by the elevation of PPI ratios at $16 \mathrm{kHz}$ ) and tinnitus (evidenced by the elevation of the gap ratio at 12.5 $\mathrm{kHz}$ ). Therefore, it is not clear whether or not the physiological changes in auditory cortical activation that were observed are driven by hearing loss, tinnitus, or other factors not tested, such as hyperacusis (Eggermont, 2012). In this case, the tinnitus behavior and hearing loss were partially separable based on sound frequency.

It is not uncommon for the tinnitus frequency to differ from the center frequency of noise trauma (Longenecker and Galazyuk, 2011) and may often be lower than the traumatic acoustic stimulus in chronic stages, especially in older animals (Wang et al., 2008). It should be noted that the frequency at which tinnitusrelated deficits were most prominent in the current study, 12.5 $\mathrm{kHz}$, is well within the bandwidth of the trauma stimulus (1 octave band centered at $16 \mathrm{kHz}$ ). There are multiple studies showing that hearing loss induced by noise or other mechanisms other than acoustic trauma (e.g., salicylate), with or without a behavioral assessment of tinnitus, can induce similar changes in the auditory cortex (Wang et al., 2006; Sarro et al., 2008; Sun et al., 2009, 2012; Xu et al., 2010a,b). In addition, a recent report demonstrates that early conductive hearing loss can lead to longlasting decreases in cortical inhibition (Takesian et al., 2012). These findings suggest that hearing loss may play a significant role in the results described here. Future studies that relate the spectral characteristics of the animals' behavior to the spectral representations in the auditory cortex will help to clarify the rel-
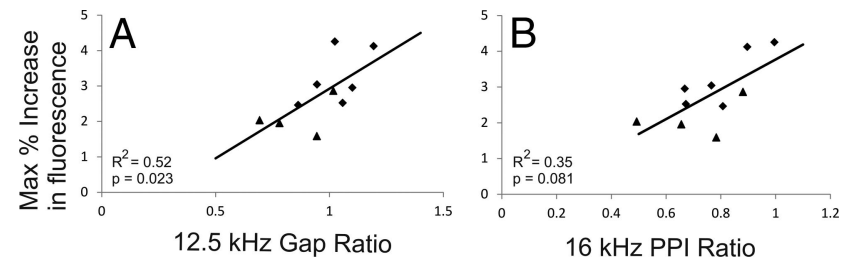

Figure 8. $\quad \boldsymbol{A}$, Correlation between maximum increase in fluorescence versus gap startle ratio at $12.5 \mathrm{kHz}$. $\boldsymbol{B}$, Correlation between maximum increase in fluorescence versus PPI ratio at 16 $\mathrm{kHz}$. Black diamonds, Noise-exposed animals; black triangles, sham-treated animals. $R^{2}$ and $p$ values generated from Spearman's correlations.

ative contributions of hearing loss versus tinnitus in changes in auditory cortical activation.

The data presented here are consistent with the hypothesis that tinnitus may be associated with cortical hyperexcitability caused by a downregulation of cortical $\mathrm{GABA}_{\mathrm{A}}$ synaptic transmission. Although a substantial body of previous work has sug- 
gested that cortical changes may be found in tinnitus (Arnold et al., 1996; Mühlnickel et al., 1998; Andersson et al., 2000), the current study, by disconnecting the auditory forebrain from its peripheral inputs, demonstrates that at least a portion of the cortical changes seen in tinnitus are generated locally. The data demonstrating that noise-exposed animals show greater activations and lower $\mathrm{GABA}_{\mathrm{A}}$ sensitivity in the off-beam regions of cortex also suggest that the cortical changes may not be restricted to a particular part of the auditory cortex and may be greater in the corticocortical projections than the thalamocortical projections. These data are consistent with previous work demonstrating diminished cortical GABAergic transmission (Wang et al., 2011), although these changes are likely dependent on the spectral content of the noise trauma and pitch of tinnitus percept (Yang et al., 2011).

GABAergic inhibition appeared sensitive to noise exposure. It is known that feedforward inhibition can critically shape cortical information processing. For example, thalamocortical projections are known to make powerful feedforward synaptic connections onto intrinsic inhibitory interneurons (Cruikshank et al., 2007; Schiff and Reyes, 2012). In addition, GABAergic interneurons are interposed between corticocortical projections, often have their synapses positioned at proximal locations on the dendritic shaft (Gonchar and Burkhalter, 1999), and therefore have significant effect on corticocortical projections (Swadlow, 2003). There are multiple types of intrinsic GABAergic neurons in the cortex (Porter et al., 2001; Dumitriu et al., 2007; Gonchar et al., 2007), and it is not known which of these may be downregulated after noise damage. It is possible that thalamocortical, corticocortical, or both may be effected by tinnitus, and the identification of the subtype of downregulated GABAergic neurons may potentially provide a specific therapeutic target for the treatment of tinnitus. Indeed, drugs that enhance $\mathrm{GABA}_{\mathrm{A}}$ ergic synaptic transmission, such as the benzodiazepines, have been shown to provide some relief for the symptoms of tinnitus but come at the cost of sedation (Bahmad et al., 2006; Darlington and Smith, 2007). Therefore, more specific targeting of subpopulations of involved neurons may prove to have therapeutic value. Other therapies that dampen synaptic transmission, such as transcranial magnetic stimulation, may also has been used to target the cortical networks underlying tinnitus (Kleinjung et al., 2008), and the mechanism for the potential efficacy of this approach may lie in the hyperexcitability described herein.

Finally, this work may shed light on the influence of aging on tinnitus. In this study, tinnitus behavior and changes in cortical responsiveness were observed well over 1 year after the noise trauma and were observed in a population of animals of an age range known to be associated with significant hearing loss (Zheng et al., 1999). In addition, previous work has suggested that aging is associated with drops in cortical GABAergic transmission (Ling et al., 2005; Caspary et al., 2008; Burianova et al., 2009; Hughes et al., 2010), such that there may be interactions between the drops in cortical $\mathrm{GABA}_{\mathrm{A}}$ ergic transmission associated with normal aging and with tinnitus. It is not known whether aging plus noise exposure depress auditory cortical GABA levels decreased beyond what would be expected from either insult on its own. However, such a hypothesized "double-hit" mechanism may help explain the high levels of tinnitus seen with aging (Møller, 2011). In addition, given the known differences in the physiology and GABA sensitivity seen in the auditory cortex with aging, it is likely that any potential new therapeutic mechanism for tinnitus may work differently in aged versus young subjects. Therefore, it will be important to use both aged and young animals in the transla- tional approaches to the development of new therapeutic modalities for tinnitus.

\section{References}

Andersson G, Lyttkens L, Hirvel ä C, Furmark T, Tillfors M, Fredrikson M (2000) Regional cerebral blood flow during tinnitus: a PET case study with lidocaine and auditory stimulation. Acta Otolaryngol 120:967-972.

Arnold W, Bartenstein P, Oestreicher E, Römer W, Schwaiger M (1996) Focal metabolic activation in the predominant left auditory cortex in patients suffering from tinnitus: a PET study with $\left[{ }^{18} \mathrm{~F}\right]$ deoxyglucose. ORL J Otorhinolaryngol Relat Spec 58:195-199.

Bahmad FM Jr, Venosa AR, Oliveira CA (2006) Benzodiazepines and GABAergics in treating severe disabling tinnitus of predominantly cochlear origin. Int Tinnitus J 12:140-144.

Broicher T, Bidmon HJ, Kamuf B, Coulon P, Gorji A, Pape HC, Speckmann EJ, Budde T (2010) Thalamic afferent activation of supragranular layers in auditory cortex in vitro: a voltage sensitive dye study. Neuroscience 165:371-385.

Brozoski TJ, Bauer CA, Caspary DM (2002) Elevated fusiform cell activity in the dorsal cochlear nucleus of chinchillas with psychophysical evidence of tinnitus. J Neurosci 22:2383-2390.

Budinger E, Heil P, Scheich H (2000) Functional organization of auditory cortex in the Mongolian gerbil (Meriones unguiculatus). III. Anatomical subdivisions and corticocortical connections. Eur J Neurosci 12:24252451.

Burianova J, Ouda L, Profant O, Syka J (2009) Age-related changes in GAD levels in the central auditory system of the rat. Exp Gerontol 44:161-169.

Caspary DM, Ling L, Turner JG, Hughes LF (2008) Inhibitory neurotransmission, plasticity and aging in the mammalian central auditory system. J Exp Biol 211:1781-1791.

Caviness VS Jr (1975) Architectonic map of neocortex of the normal mouse. J Comp Neurol 164:247-263.

Cruikshank SJ, Killackey HP, Metherate R (2001) Parvalbumin and calbindin are differentially distributed within primary and secondary subregions of the mouse auditory forebrain. Neuroscience 105:553-569.

Cruikshank SJ, Rose HJ, Metherate R (2002) Auditory thalamocortical synaptic transmission in vitro. J Neurophysiol 87:361-384.

Cruikshank SJ, Lewis TJ, Connors BW (2007) Synaptic basis for intense thalamocortical activation of feedforward inhibitory cells in neocortex. Nat Neurosci 10:462-468.

Darlington CL, Smith PF (2007) Drug treatments for tinnitus. In: Progress in brain research (Langguth B, Hajak G, Kleinjung T, Cacace A, Møller AR, eds), pp 249-262. Amsterdam: Elsevier.

Dumitriu D, Cossart R, Huang J, Yuste R (2007) Correlation between axonal morphologies and synaptic input kinetics of interneurons from mouse visual cortex. Cereb Cortex 17:81-91.

Eggermont JJ (2007) Pathophysiology of tinnitus. In: Progress in brain research (Langguth B, Hajak G, Kleinjung T, Cacace A, Møller AR, eds), pp 19-543. Amsterdam: Elsevier.

Eggermont JJ (2012) Hearing loss, hyperacusis, or tinnitus: what is modeled in animal research? Hear Res. Advanced online publication. Retrieved September 28, 2012. doi:10.1016/j.heares.2012.01.005.

Franklin K, Paxinos G (2007) The mouse brain in stereotaxic coordinates, Ed 3. Amsterdam: Elsevier Science.

Gonchar Y, Burkhalter A (1999) Differential subcellular localization of forward and feedback interareal inputs to parvalbumin expressing GABAergic neurons in rat visual cortex. J Comp Neurol 406:346-360.

Gonchar Y, Wang Q, Burkhalter AH (2007) Multiple distinct subtypes of GABAergic neurons in mouse visual cortex identified by triple immunostaining. Front Neuroanat 1:3.

Hackett TA, Barkat TR, O’Brien BM, Hensch TK, Polley DB (2011) Linking topography to tonotopy in the mouse auditory thalamocortical circuit. J Neurosci 31:2983-2995.

Hughes LF, Turner JG, Parrish JL, Caspary DM (2010) Processing of broadband stimuli across A1 layers in young and aged rats. Hear Res 264:79-85.

Husson TR, Mallik AK, Zhang JX, Issa NP (2007) Functional imaging of primary visual cortex using flavoprotein autofluorescence. J Neurosci 27:8665-8675.

Hwang JH, Chen JC, Yang SY, Wang MF, Liu TC, Chan YC (2011) Expression of COX-2 and NMDA receptor genes at the cochlea and midbrain in salicylate-induced tinnitus. Laryngoscope 121:361-364. 
Kaltenbach JA (2011) Tinnitus: models and mechanisms. Hear Res 276:52-60.

Kaltenbach JA, Godfrey DA, Neumann JB, McCaslin DL, Afman CE, Zhang J (1998) Changes in spontaneous neural activity in the dorsal cochlear nucleus following exposure to intense sound: relation to threshold shift. Hear Res 124:78-84.

Kleinjung T, Vielsmeier V, Landgrebe M, Hajak G, Langguth B (2008) Transcranial magnetic stimulation: a new diagnostic and therapeutic tool for tinnitus patients. Int Tinnitus J 14:112-118.

Ling LL, Hughes LF, Caspary DM (2005) Age-related loss of the GABA synthetic enzyme glutamic acid decarboxylase in rat primary auditory cortex. Neuroscience 132:1103-1113.

Llano DA, Sherman SM (2008) Evidence for nonreciprocal organization of the mouse auditory thalamocortical-corticothalamic projection systems. J Comp Neurol 507:1209-1227.

Llano DA, Theyel BB, Mallik AK, Sherman SM, Issa NP (2009) Rapid and sensitive mapping of long-range connections in vitro using flavoprotein autofluorescence imaging combined with laser photostimulation. J Neurophysiol 101:3325-3340.

Lobarinas E, Yang G, Sun W, Ding D, Mirza N, Dalby-Brown W, Hilczmayer E, Fitzgerald S, Zhang L, Salvi R (2006) Salicylate- and quinine-induced tinnitus and effects of memantine. Acta Otolaryngol Suppl (556):13-19.

Longenecker RJ, Galazyuk AV (2011) Development of tinnitus in CBA/CAj mice following sound exposure. J Assoc Res Otolaryngol 12:647-658.

Middleton JW, Kiritani T, Pedersen C, Turner JG, Shepherd GM, Tzounopoulos T (2011) Mice with behavioral evidence of tinnitus exhibit dorsal cochlear nucleus hyperactivity because of decreased GABAergic inhibition. Proc Natl Acad Sci U S A 108:7601-7606.

Molinari M, Dell'Anna ME, Rausell E, Leggio MG, Hashikawa T, Jones EG (1995) Auditory thalamocortical pathways defined in monkeys by calciumbinding protein immunoreactivity. J Comp Neurol 362:171-194.

Møller AR (2011) Epidemiology of tinnitus in adults. In: Textbook of tinnitus (Møller AR, Langguth B, Ridder D, Kleinjung T), pp 29-37. New York: Springer.

Mühlnickel W, Elbert T, Taub E, Flor H (1998) Reorganization of auditory cortex in tinnitus. Proc Natl Acad Sci U S A 95:10340-10343.

Noreña AJ, Moffat G, Blanc JL, Pezard L, Cazals Y (2010) Neural changes in the auditory cortex of awake guinea pigs after two tinnitus inducers: salicylate and acoustic trauma. Neuroscience 166:1194-1209.

Porter JT, Johnson CK, Agmon A (2001) Diverse types of interneurons generate thalamus-evoked feedforward inhibition in the mouse barrel cortex. J Neurosci 21:2699-2710.

Reinert KC, Gao W, Chen G, Wang X, Peng YP, Ebner TJ (2011) Cellular and metabolic origins of flavoprotein autofluorescence in the cerebellar cortex in vivo. Cerebellum 10:585-599.

Salvi RJ, Saunders SS, Gratton MA, Arehole S, Powers N (1990) Enhanced evoked response amplitudes in the inferior colliculus of the chinchilla following acoustic trauma. Hear Res 50:245-257.

Sarro EC, Kotak VC, Sanes DH, Aoki C (2008) Hearing loss alters the subcellular distribution of presynaptic GAD and postsynaptic GABAA receptors in the auditory cortex. Cereb Cortex 18:2855-2867.

Schecklmann M, Landgrebe M, Poeppl TB, Kreuzer P, Männer P, Marienhagen J, Wack DS, Kleinjung T, Hajak G, Langguth B (2011) Neural correlates of tinnitus duration and distress: a positron emission tomography study. Hum Brain Mapp. Advanced online publication. Retrieved September 28, 2012.

Schiff ML, Reyes AD (2012) Characterization of thalamocortical responses of regular-spiking and fast-spiking neurons of the mouse auditory cortex in vitro and in silico. J Neurophysiol 107:1476-1488.

Shibuki K, Hishida R, Murakami H, Kudoh M, Kawaguchi T, Watanabe M, Watanabe S, Kouuchi T, Tanaka R (2003) Dynamic imaging of somatosensory cortical activity in the rat visualized by flavoprotein autofluorescence. J Physiol 549:919-927.
Stolzberg D, Chen GD, Allman BL, Salvi RJ (2011) Salicylate-induced peripheral auditory changes and tonotopic reorganization of auditory cortex. Neuroscience 180:157-164.

Sun W, Lu J, Stolzberg D, Gray L, Deng A, Lobarinas E, Salvi RJ (2009) Salicylate increases the gain of the central auditory system. Neuroscience 159:325-334.

Sun W, Deng A, Jayaram A, Gibson B (2012) Noise exposure enhances auditory cortex responses related to hyperacusis behavior. Brain Res. Advance online publication. Retrieved September 28, 2012. doi:10.1016/j.brainres.2012.02.008.

Swadlow HA (2003) Fast-spike interneurons and feedforward inhibition in awake sensory neocortex. Cereb Cortex 13:25-32.

Szczepaniak WS, Møller AR (1995) Evidence of decreased GABAergic influence on temporal integration in the inferior colliculus following acute noise exposure: a study of evoked potentials in the rat. Neurosci Lett 196:77-80.

Takahashi K, Hishida R, Kubota Y, Kudoh M, Takahashi S, Shibuki K (2006) Transcranial fluorescence imaging of auditory cortical plasticity regulated by acoustic environments in mice. Eur J Neurosci 23:1365-1376.

Takesian AE, Kotak VC, Sanes DH (2012) Age-dependent effect of hearing loss on cortical inhibitory synapse function. J Neurophysiol 107:937-947.

Turner J (2007) Behavioral measures of tinnitus in laboratory animals. In: Progress in brain research (Langguth B, Hajak G, Kleinjung T, Cacace A, Møller AR, eds), pp 147-549. Amsterdam: Elsevier.

Turner J, Larsen D, Hughes L, Moechars D, Shore S (2012) Time course of tinnitus development following noise exposure in mice. J Neurosci Res 90:1480-1488.

Turner JG, Brozoski TJ, Bauer CA, Parrish JL, Myers K, Hughes LF, Caspary DM (2006) Gap detection deficits in rats with tinnitus: a potential novel screening tool. Behav Neurosci 120:188-195.

van der Loo E, Gais S, Congedo M, Vanneste S, Plazier M, Menovsky T, Van de Heyning P, De Ridder D (2009) Tinnitus intensity dependent gamma oscillations of the contralateral auditory cortex. PLoS One 4:e7396.

Wallace MN, Kitzes LM, Jones EG (1991) Chemoarchitectonic organization of the cat primary auditory cortex. Exp Brain Res 86:518-526.

Wang H, Ling L, Brozoski T, Turner J, Parrish J, Hughes LF, Caspary DM (2008) Altered markers of glycinergic function in the DCN of a rat model of tinnitus. Assoc Res Otolaryngol 31:117.

Wang H, Brozoski TJ, Caspary DM (2011) Inhibitory neurotransmission in animal models of tinnitus: maladaptive plasticity. Hear Res 279:111-117.

Wang HT, Luo B, Zhou KQ, Xu TL, Chen L (2006) Sodium salicylate reduces inhibitory postsynaptic currents in neurons of rat auditory cortex. Hear Res 215:77-83.

Willott JF, Lu SM (1982) Noise-induced hearing loss can alter neural coding and increase excitability in the central nervous system. Science 216:1331-1334.

Wong-Riley M (1979) Changes in the visual system of monocularly sutured or enucleated cats demonstrable with cytochrome oxidase histochemistry. Brain Res 171:11-28.

Xu J, Yu L, Cai R, Zhang J, Sun X (2010a) Early continuous white noise exposure alters auditory spatial sensitivity and expression of GAD65 and GABAA receptor subunits in rat auditory cortex. Cereb Cortex 20:804-812.

Xu J, Yu L, Zhang J, Cai R, Sun X (2010b) Early continuous white noise exposure alters 1- $\alpha$-amino-3-hydroxy-5-methyl-4-isoxazole propionic acid receptor subunit glutamate receptor 2 and $\gamma$-aminobutyric acid type a receptor subunit $\beta 3$ protein expression in rat auditory cortex. J Neurosci Res 88:614-619.

Yang S, Weiner BD, Zhang LS, Cho SJ, Bao S (2011) Homeostatic plasticity drives tinnitus perception in an animal model. Proc Natl Acad Sci U S A 108:14974-14979.

Zheng QY, Johnson KR, Erway LC (1999) Assessment of hearing in 80 inbred strains of mice by ABR threshold analyses. Hear Res 130:94-107. 